\title{
Erstlinientherapie mit Cetuximab kann das Überleben verlängern
}

Die Erstlinientherapie hat für das Gesamtüberleben von Patienten mit metastasiertem kolorektalen Karzinom (mCRC) eine besondere Bedeutung, so Prof. Dr. Claus-Henning Köhne, Oldenburg. Daher sei in der Erstliniensituation der Einsatz einer intensiven Kombinationstherapie, wie beispielsweise eine Kombinationschemotherapie plus EGFR (Epidermal Growth Factor Receptor)-Antikörper, unter Berücksichtigung von Toxizität und Überlebenswahrscheinlichkeit für alle mCRC-Patienten, d.h. auch asymptomatische Patienten mit KRASWildtyp-Tumor, zu überlegen. Diese Einschätzung unterstützen die Ergebnisse der CRYSTAL-Studie, in der bei mCRC-Patienten mit KRAS-Wildtyp-Tumor unter FOLFIRI plus Cetuximab im Vergleich zur alleinigen Chemotherapie ein Überlebensvorteil von median fast vier Monaten beobachtet wurde.

Beim mCRC werden unter Berücksichtigung des primären Behandlungsziels drei Patientengruppen unterschieden: Gruppe 1 umfasst Patienten mit initial nicht resezierbaren Lebermetastasen, bei denen die Resezierbarkeit angestrebt wird, in Gruppe 2 sind Patienten mit nicht resezierbaren Metastasen, hoher Tumorlast oder tumorbedingten Symptomen zusammengefasst, bei denen primär eine Symptomkontrolle notwendig ist, und Gruppe 3 schließt $\mathrm{Pa}$ tienten mit definitiv nicht resezierbaren Metastasen und asymptomatischem oder weniger aggressivem Verlauf ein, bei denen die Lebensverlängerung und der Erhalt der Lebensqualität Priorität hat.

Aus dieser Einteilung leitet sich die Intensität der Therapie ab. Patienten der Gruppen 1 und 2 werden zumeist intensiv behandelt. Wie intensiv Patienten der Gruppe $3 \mathrm{zu}$ behandeln sind, wird häufig diskutiert. Hilf- reich für die Entscheidungsfindung und einen personalisierten Ansatz ist für Köhne das STEP-Schema, bei dem es sehr wichtig ist, die Strategie der Behandlung frühzeitig zu definieren (kurativer versus palliativer Ansatz) sowie die Tumorbiologie (aggressiv versus indolent), eine eventuelle EGFR-Abhängigkeit des Tumorwachstums (KRAS Wildtyp versus mutiertes KRAS-Gen) und nicht zuletzt die Sorgen und Wünsche der Patienten zu berücksichtigen.

\section{Intensive Therapie mit EGFR- Antikörper bei KRAS-Wildtyp}

Liegt ein KRAS-Wildtyp-Tumor vor, können mCRC-Patienten aller drei Gruppen von einer intensiven Therapie mit FOLFIRI (Folinsäure/5-Fluorouracil [5-FU]/Irinotecan) plus Cetuximab (Erbitux ${ }^{\varpi}$ ) profitieren, wie in der Studie CRYSTAL (Cetuximab combined with Irinotecan in first-line The-

\section{Metastasiertes oder lokal fortgeschrittenes Mammakarzinom Überlebensvorteil durch Eribulin}

Wie die Ergebnisse der zulassungsrelevanten Phase-III-Studie EMBRACE (Eisai Metastatic Breast Cancer Study Assessing Physician's Choice vs. Eribulin) belegen, ist Eribulin (Halaven ${ }^{\oplus}$ ) die erste Monochemotherapie, mit der im Vergleich zu einer Behandlung nach Wahl des Arztes ein deutlicher, klinisch relevanter Überlebensvorteil bei Patientinnen mit stark vorbehandeltem und fortgeschrittenem Mammakarzinom erreicht werden kann (Cortes J et al., Lancet 2011, 377:914-923). Daten von Post-hoc-
Analysen zufolge ist der Gesamtüberlebensvorteil umso größer, je weniger Vorbehandlungen die Patientinnen erhalten hatten (Twelves C et al., SABCS 2010:\#P6, 14-08).

Die Arbeitsgemeinschaft Gynäkologische Onkologie e.V. (AGO) empfiehlt Eribulin in ihren Leitlinien als eine Therapie der ersten Wahl bei Patientinnen mit metastasiertem Mammakarzinom nach Taxan- und Anthrazyklinvorbehandlung (www.ago-online.de).

nach Informationen der Eisai GmbH rapy for Metastatic colorectal Cancer) gezeigt wurde (van Cutsem E et al., N Engl J Med 2009, 360:1408-1417; van Cutsem E et al., J Clin Oncol 2011, 29:2011-2019). Im Vergleich zur alleinigen Chemotherapie wurden durch die intensive Kombinationstherapie mit Cetuximab das Ansprechen ( $40 \%$ versus $57 \%$; $\mathrm{p}<0,001)$, das progressionsfreie Überleben (median 8,4 versus 9,9 Monate; $p=0,0012$ ) und das Gesamtüberleben (median 20,0 versus 23,5 Monate; $\mathrm{p}=0,0093$ ) signifikant verbessert.

\section{Längeres Überleben bei früher \\ Tumorschrumpfung}

Dabei profitierten Patienten mit früher $\mathrm{Tu}-$ morschrumpfung ganz besonders hinsichtlich des Gesamtüberlebens, wie Prof. Dr. Dirk Arnold, Hamburg, anhand der Ergebnisse einer retrospektiven Analyse zeigte (Piessevaux H et al., Ann Oncol 2010, 21 [Suppl 8]:\#596P). Bei Patienten mit einer Tumor-bzw. Metastasengrößenreduktion um $\geq 20 \%$ in Woche 8 betrug das mediane Gesamtüberleben unter FOLFIRI plus Cetuximab in der CRYSTAL-Studie 30,0 Monate versus 18,6 Monate bei Patienten, die unter der Kombinationstherapie mit dem EGFRAntikörper zu diesem Zeitpunkt nur eine Tumor- bzw. Metastasengrößenreduktion um $<20 \%$ hatten $(p<0,001)$. In der Studie OPUS (Oxaliplatin and Cetuximab in firstline Treatment of $\mathrm{mCRC}$ ) bestätigte sich dieser Zusammenhang unter FOLFOX (Folinsäure/5-FU/Oxaliplatin) plus Cetuximab mit einem medianen Gesamtüberleben von 26,0 Monaten versus 15,6 Monate $(\mathrm{p}=0,006)$.

\section{Ansprechen für Patientennutzen relevant}

Eine schnelle deutliche Tumor- bzw. Metastasengrößenreduktion hat laut Arnold auch eine besondere Qualität im Patientennutzen. Für Patienten der Gruppe 2 bedeutet dies zum Beispiel eine schnelle Symptomlinderung. In der CRYSTAL-Studie war die maximale Symptomlinderung unter FOLFIRI plus Cetuximab bereits nach acht Wochen zu beobachten versus 16 Wochen unter alleiniger Chemotherapie (Griebsch I et al., ASCO 2011:\#3626). (am)

Satellitensymposium der Merck Serono GmbH 